\title{
Capsule Commentary on Dolan et al., The Effectiveness of a Physician-Only and Physician-Patient Intervention on Colorectal Cancer Screening Discussions Between Providers and African American and Latino Patients
}

\author{
Victor O. Kolade, MD \\ Department of Medicine, The Guthrie Clinic, Sayre, PA, USA.
}

J Gen Intern Med 30(12): 1848

DOI: $10.1007 / \mathrm{s} 11606-015-3461-9$

(c) Society of General Internal Medicine 2015

$\mathrm{C}$ olorectal cancer (CRC) is one of the most prevalent cancers in the United States, and has several screening options. Unfortunately, screening rates have been suboptimal for years. ${ }^{1}$ For most screening modalities, colon preparation is not endearing, and screening is not yet fully reimbursed by insurance without provider referrals, as is the case for Pap smears, or without a provider visit at all, as with some nicotine replacement therapies.

This study sought to evaluate the usefulness of patient education via video compared to physician education as a means of enhancing screening among patients at federally qualified health centers or academic medical centers. ${ }^{2}$

The 'waiting room video' is an attractive way to use patient downtime before an appointment, and is available in both English and Spanish. Providing information directly to patients matches the intent of the Ecological Model for Ambulatory Patient Safety in Chronic Disease to produce collaboration between an activated patient and a proactive practice team. ${ }^{3}$

An important limitation is the failure of clinic randomization, making generalization more difficult. It is also not clear whether medical residents always saw patients with the same attending; sub-total concordance may have reduced the efficiency of physician randomization, which was done by attending. Since non-white Americans have lower rates of CRC screening, ${ }^{4}$ it is a study strength that all patients were either Non-Hispanic Black or Hispanic/Latino.

Why were colonoscopy discussions more popular than other screening modalities? This test may be preferred by patients, as it does not require user contact with fecal samples.
Providers may prefer it because it has the longest 'shelf-life'up to 10 years - and is required to address abnormalities on the other tests. Unfortunately, many of the study patients may lack access to colonoscopy. Alternatively, high rates of fecal occult blood testing can be obtained in low-income populations. ${ }^{5}$ This study focused on discussions rather than actual rates of CRC screening.

What can be done next? Pre-visit videos may be more effective if provided via electronic medical record portals or the internet earlier than at the hour of the visit. Studies of the effect of increasing screening discussions on actual screening rates are needed.

Conflicts of Interest: The author has no conflicts of interest with this article.

Corresponding Author: Victor O. Kolade, MD; Department of MedicineThe Guthrie Clinic, 1 Guthrie Square, Sayre, PA 18840, USA (e-mail: VKOLADE@gmail.com).

\section{REFERENCES}

1. Centers for Disease Control and Prevention. Vital signs: colorectal cancer screening, incidence, and mortality-United States, 2002-2010. MMWR Morb Mortal Wkly Rep. 2011;60(26):884-889.

2. Dolan NC, Ramirez-Zohfeld V, Rademaker AQ, Ferreira MR, Galanter WL, Radosta J, Eder M, Cameron KA. The effectiveness of a physician-only and physician-patient intervention on colorectal cancer screening discussions between providers and African American and Latino patients. J Gen Intern Med. 2015. doi:10.1007/s11606-015-3381-8.

3. Sarkar U, Wachter RM, Schroeder SA, Schillinger D. Refocusing the lens: patient safety in ambulatory chronic disease care. Jt Comm J Qual Patient Saf. 2009;35(7):377-383.

4. Ananthakrishnan AN, Schellhase KG, Sparapani RA, Laud PW, Neuner JM. Disparities in colon cancer screening in the Medicare population. Arch Intern Med. 2007;167(3):258-264.

5. Baker DW, Brown T, Buchanan DR, Weil J, Balsley K, Ranalli L, et al. Comparative Effectiveness of a Multifaceted Intervention to Improve Adherence to Annual Colorectal Cancer Screening in Community Health Centers. JAMA Intern Med. 2014;174(8):1235-1241. 